PROCEEDINGS OF THE

AMERICAN MATHEMATICAL SOCIETY

Volume 130, Number 8, Pages 2311-2312

S 0002-9939(02)06540-1

Article electronically published on March 13, 2002

\title{
A GEOMETRIC PROOF OF THE EXISTENCE OF THE GREEN BUNDLES
}

\author{
RENATO ITURRIAGA
}

(Communicated by Michael Handel)

Abstract. We give a new proof of the existence of the Green bundles.

Let $(M, g)$ be a compact Riemannian manifold, and denote by $g_{t}$ its geodesic flow on the tangent bundle $T M$. Let $\pi: T M \rightarrow M$ be the canonical projection and for all $\theta \in T M$ let $V(\theta)$ be the kernel of $d \pi_{\theta}$. Two points $\theta_{1}, \theta_{2}$ are said to be conjugate if $\theta_{2}=g_{t} \theta_{1}$ and $d g_{t} V\left(\theta_{1}\right) \cap V\left(\theta_{2}\right) \neq 0$.

It was proved by Hopf [5] that a two-dimensional torus without conjugate points is flat. Afterwards, Green [8] proved that the integral of the scalar curvature of a manifold without conjugate points is nonpositive and it vanishes if the metric is locally flat. A main ingredient was the existence, under the condition of no conjugate points, of the following bundles:

$$
\begin{aligned}
& E^{s}(\theta)=\lim _{t \rightarrow \infty} d g_{-t} V\left(g_{t}(\theta)\right), \\
& E^{u}(\theta)=\lim _{t \rightarrow \infty} d g_{t} V\left(g_{-t}(\theta)\right) .
\end{aligned}
$$

Hopf's result was generalized to higher dimensions in [2], but there are still new rigidity type results using these bundles; see for example [1].

These bundles have other applications: among other ideas they where used by Freire and Mañé [7] to obtain estimates of the topological entropy. Foulon [6] generalized this result to the case of Finsler metrics. The bundles were also used by Eberlain [4] who proved that these are transverse if and only if the geodesic flow is Anosov. This result was also generalized to the case of convex Hamiltonians without conjugate points; see [3]. The purpose of this note is to give a new proof of the following

Theorem. If the geodesic flow $g_{t}$ of a compact manifold does not have conjugate points, then for every $\theta$ in TM the limits (11) and (2) exist.

We recall from [4] the definition of the connection map $K: T_{\theta} T M \rightarrow T_{\pi(\theta)} M$. For $\xi$ on $T_{\theta} T M$ let $Z:(-\epsilon, \epsilon) \rightarrow T M$ be a curve with initial velocity $\xi$. Define $K(\xi)=Z^{\prime}(0)$ to be the covariant derivative of $Z$ along the curve $\pi \circ Z$. The definition does not depend on the curve $Z$.

Received by the editors February 8, 2001.

2000 Mathematics Subject Classification. Primary 37D40.

The author was partially supported by CONACYT-México grant \#28489-E and EPSRCUnited Kingdom GR/M5610. 
Denote $E_{T}=d g_{-T} V\left(g_{T}(\theta)\right)$. Since $d \pi: E_{T}(\theta) \rightarrow T_{\pi(\theta))} M$ is an isomorphism, we may use the connection map to define a linear map $S_{T}: T_{\pi(\theta)} M \rightarrow T_{\pi(\theta)} M$ by $S_{T}(Y)=K(\xi)$ where $\xi$ is in $E_{T}$ and $Y=d \pi(\xi)$.

Let $\omega$ be the canonical symplectic structure on $T M$, i.e. $\omega(\xi, \eta)=\langle d \pi(\xi), K(\eta)\rangle-$ $\langle K(\xi), d \pi(\eta)\rangle$. Clearly the vertical space is Lagrangian, meaning that $\omega(\xi, \eta)=0$ for all $\xi, \eta$ on $V(\theta)$. Since the geodesic flow preserves the symplectic form, it follows that $E_{T}$ is a Lagrangian subspace. This immediately implies that $S_{T}$ is a symmetric linear map.

Recall that it is possible to give a partial order in the set of symmetric linear transformations defining $A \succ B$ if $A-B$ is positive definite. To prove the theorem it is enough to see that the sequence $S_{T}$ is monotone and bounded. The original proof of this fact is a slightly clumsy calculation.

For all $t<s$ the linear transformation $S_{s}-S_{t}$ is symmetric. We claim that the signature does not change in the region contained in $\mathbb{R}^{2}$ such that $0<t<s$. Otherwise we can find $t_{0}<s_{0}$ such that $S_{s_{0}}-S_{t_{0}}$ has 0 as an eigenvalue; this implies that $\theta_{s_{0}}$ and $\theta_{t_{0}}$ are conjugate.

On the other hand let $Y_{\xi}(t)$ be the Jacobi field along the geodesic $\gamma=\pi g_{t}$ with initial conditions $Y(0)=d \pi(\xi)$ and $Y^{\prime}(0)=K(\xi)$. Denote by $P_{-t}: T_{\gamma(t)} M \rightarrow$ $T_{\pi(\theta)} M$ the parallel translation. Then for $\xi$ in $E_{s}(\theta)$ we have that

$$
0=P_{-s}\left(Y_{\xi}(s)\right)=Y_{\xi}(0)+s Y_{\xi}^{\prime}(0)+O\left(s^{2}\right)=\left(I d+s S_{s}\right)\left(Y_{\xi}(0)\right),
$$

where $I d$ is the identity transformation. Hence $S_{s}=-\frac{1}{s} I d+O\left(s^{2}\right)$. Consequently $S_{s}-S_{t}$ is positive definite and $S_{s}$ is monotone increasing as $s$ tends to infinity.

Similarly for all $t<0<s$ the linear transformation $S_{s}-S_{t}$ has constant signature. Moreover by the same estimate the linear transformation $S_{s}-S_{t}$ is negative definite, for $|s|,|t| \ll 1$. So $S_{s}$ is bounded by $S_{t}$ for any $t<0$. Similar arguments apply for the unstable bundle $E^{u}$.

Finally let us remark that the same argument, the constancy of the signature of $S_{s}-S_{t}$ and a local computation applies for convex Hamiltonians without conjugate points. See $[3$ to define the order of symmetric matrices,

\section{REFERENCES}

[1] M. L. Bialy, Rigidity for periodic magnetic fields, Ergodic Th. and Dynam. Sys. 20 (2000), 1619-1626. CMP 2001:06

[2] D. Burago, S. Ivanov, Riemannian Tori without conjugate points are flat, GAFA (1994), 259-269. MR 95h:53049

[3] G. Contreras, R. Iturriaga, Convex Hamiltonians without conjugate points, Ergod. Th. and Dynam. Sys. 19 (1999), 901-952. MR 2000h:37102

[4] P. Eberlain, When is a geodesic flow af Anosov type? I, J. Differential Geometry 8 (1973), 437-463. MR 52:1788

[5] E. Hopf, Closed surfaces without conjugate points, Proc. Natl. Acad. Sci 34 (1948), 47-51. MR 9:378d

[6] P. Foulon, Estimation de l'entropie des systemes lagrangiens sans points conjugués, Ann. Inst. Henri Poincare 57 (1992), 117-146. MR 93k:58178

[7] A. Freire, R. Mañé, On the entropy of the geodesic flow of manifolds without conjugate points, Inv. Math. 69 (1982), 375-392. MR 84d:58063

[8] L.W. Green, A theorem of E. Hopf, Michigan Math. J. 5 (1958), 31-34. MR 20:4300

Cimat, A.P. 402, 36000 Guanajuato, Gto, México - and - I. Newton Institute, CamBRIDGE UNIVERSITY

E-mail address: renato@fractal.cimat.mx 